\title{
First observations on reproductive characteristics on Chilean Patagonian populations inland water Calanoids Copepods
}

\section{Primeras observaciones en características reproductivas en poblaciones chilenas de Copépodos Calanoideos de aguas continentales}

\author{
Patricio De los Rios-Escalante ${ }^{1}$ \& Eriko Carreño ${ }^{2}$ \\ ${ }^{1}$ Universidad Católica de Temuco, Facultad de Recursos Naturales, Escuela de Ciencias Ambientales, Casilla 15-D, Temuco. \\ ${ }^{2}$ Universidad Católica de Temuco, Facultad de Recursos Naturales, Escuela de Acuicultura, Casilla 15-D, Temuco.
}

\begin{abstract}
RESUMEN
Los copépodos calanoideos son abundantes en los ensambles zooplanctónicos de aguas continentales de la Patagonia, y en este contexto, se analizaron parámetros de fertilidad en cuatro especies de calanoideos representativos de aguas continentales de la Patagonia $\left(37^{\circ}-52^{\circ} \mathrm{S}\right)$ con el objetivo de determinar los parámetros de fertilidad en poblaciones de copépodos representativas de la Patagonia chilena. Para este propósito se consideraron dos a cuatro poblaciones de las siguientes especies: Boeckella gracilipes, B. michaelseni, B. poppei, y Tumeodiaptomus diabolicus, se consideraron individuos hembras con huevos a las que se midió el largo total, número de huevos y la razón entre número de huevos con el largo total. Los resultados mostraron que a nivel de especies $B$. poppei fueron significativamente diferentes para las otras tres especies. A nivel de poblaciones, se encontraron diferencias a nivel de poblaciones en algunas especies. Los resultados que los parámetros reproductivos variaron en función de los parámetros ambientales variaron en función de las condiciones ambientales de las poblaciones y estaría asociado con las condiciones ecológicas tales como estructuras poblacionales y las propiedades de las comunidades zooplanctónicas.
\end{abstract}

Palabras clave: copépodos, zooplankton, lagos Patagónicos.

\begin{abstract}
The calanoids copepods are abundant in zooplankton assemblages in Patagonian inland waters, and within context the aim of determine the fertility parameters on populations of copepods representative of different inland water of Chilean Patagonia $\left(37^{\circ}-52^{\circ} \mathrm{S}\right)$. For this purpose, it was considered two to four populations of the the following species: Boeckella gracilipes, B. michaelseni, B. poppei, and Tumeodiaptomus diabolicus, it was considered for female individuals the total length, eggs number, and ratio between total length. The results revealed at species level that $B$. poppei was significantly different in comparison to another species in all of the three species. At populations level it was found differences in three species considered. The results revealed that the reproductive parameters are variable in function of the environmental conditions of the populations. The results denoted that the reproductive parameters are markedly influenced by environmental conditions, and it would be associated with ecological consequences such as populational structures and properties of zooplankton community ecology.
\end{abstract}

Keywords: copepods, zooplankton, Patagonian lakes.

\section{INTRODUCCION}

Los copépodos calanoideos son un componente importante en el zooplancton en ambientes marinos (Kiorboe 1997) y algunos ambientes lacustres como se ha reportado en la Patagonia (De los Ríos-Escalante 2010), llegando a tener una abundancia entre un 50 y $90 \%$ (Hirsk \& Bunker 2003; De los Ríos-Escalante 2010) llegando a tener una gran importancia en la estructura pelágica, en la composición comunitaria, tramas tróficas y en la productividad secundaria (Ara 2004, Daly et al. 2004).
$\mathrm{Si}$ consideramos la importancia ecológica de los copépodos en el zooplancton, ha sido necesario estudiar la productividad secundaria con parámetros del ciclo de vida, específicacmente fecundidad y características asociadas (Kiorboe 1997) la que se define como el número de huevos por individuo hembra, que junto a otros parámetros como producción, crecimiento, alimentación y mortalidad son importantes para estimar la productividad secundaria y reclutamiento entre diferentes especies y poblaciones (Hernández-Trujillo et al. 1998). La fecundidad es regulada por factores amnbientales abióticos como movimientos del 
agua, temperatura y características químicas (HernandezTrujillo et al. 2008), exposición a predadores (Gliwicz 2003), y disponibilidad de alimentpo (Ara 2004; HernándezTrujillo et al. 2008). Si consideramos estos antecedentes, los copépodos calanoideos son dominantes en lagos chilenos que son oligotróficas y monomícticos cálidos y algunas pozas temporales, no obstante no hay estudios detallados sobre dinámica poblacional (De los Ríos-Escalante 2010). El objetivo del presente estudio fue analizar la fecundidad en número de huevos por individuo hembra y su relación con el largo total en copépodos lacustres de la Patagonia chilena.

\section{MATERIALES Y METODOS}

Se consideraron especímenes hembras de las especies Boeckella gracilipes (Daday, 1902), B. michaelseni (Mrázek, 1901), B. poppei (Mrázek, 1901) y Tumeodiaptomus diabolicus (Brehm, 1939) (Tabla 1), estos especímenes se colectaron entre los años 2000 y 2004 y se fijaron con etanol absoluto. A 30 individuos se les midió largo total, número de huevos y la razón entre el largo total y el número de huevos. A los datos disponibles se les ordenó considerando primero determinar la existencia de potenciales diferencias entre especies y entre las diferentes poblaciones de cada especie. A los datos disponibles se les aplicó una prueba no paramétrica de Kruskall Wallis con la respectiva prueba de comparaciones múltiples de Dunns, para los datos en que se tuvo que comparar dos grupos, se aplicó una prueba no paramétrica U-Mann Witney, para todos los análisis estadísticos se usó el programa Graph Pad Prism 5.0.

\section{RESULTADOS}

Los resultados revelaron que el largo total varió entre 0.6 y $1.02 \mathrm{~mm}$ para las especies de lagos y lagunas $(B$. gracilipes, $B$. michaelseni y $T$. diabolicus), mientras que la especie $B$. poppei varió entre 1.60 y $2.30 \mathrm{~mm}$ (Tabla 1). El número de huevos varió entre 7.70 y 12.00 para las especies de lagos y lagunas (B. gracilipes, B. michaelseni and $T$. diabolicus), mientras que $B$. poppei varió entre 22.00 y 40.00 (Tabla 1). Finalmente, la razón entre largo total y número de huevos varió entre 0.08 y 0.19 para especies de lagos y lagunas (B. gracilipes, B. michaelseni y $T$. diabolicus), mientras que para $B$. poppei varió entre 0.07 y 0.09 (Tabla 1). Los resultados del análisis estadístico revelaron que el largo total fue significativamente alto para todas las especies y solo $B$. gracilipes y $T$. diabolicus no fueron significativamente diferentes (Tabla 2). Finalmente no hubo diferencias para las poblaciones de la especie B.poppei en comparación a las otras poblaciones (Tabla
2). Los resultados sobre diferencias entre poblaciones de una especie revelaron que para $B$. michaelseni la población del lago Riesco fue significativamente más alta, mientras que no hubo diferencias entre las poblaciones de $B$. gracilipes (Tabla 2). Para T. diabolicus solo la población del lago Calafquén fue significativamente más alta que las otras poblaciones (Tabla 2). Respecto al número de huevos, no hubo diferencia signficativa entre especies y no hubo diferencia en las poblaciones estudiadas de $B$. poppei (Tabla 2). Los resultados entre especies para $B$. michaelseni revelaron que la población del lago Riesco fue significativamente diferente en comparacion a ls otras dos poblaciones (Tabla 2). No hubo diferencias en las dos poblaciones estudiadas de $B$. gracilipes (Tabla 2). Para $T$. diabolicus solo hubo diferencias en la población del lago Calafquén (Tabla 2). Finalmente, no hubo diferencias en la poblaciones de $B$. poppei (Tabla 2). Finalmente respecto a la razón entre largo total y número de huevos fue significativamente difernte excepto para B. michaelseni y $T$. diabolicus (Tabla 2). Los resultados de comparación entre poblaciones de una especie revelaron que para $B$. michaelseni hubo diferencias entre las poblaciones de Foitzick y Riesco (Tabla 2). No hubo diferencias entre las poblaciones de $B$. gracilipes (Tabla 2). Para $T$. diabolicus solo hubo diferencias entre las poblaciones de Trapén y Calafquén respecto a la población de Lanalhue (Tabla 2). Finalmente no hubo diferencias entre las poblaciones de $B$. poppei (Tabla 2).

\section{DISCUSION}

Los resultados revelaron que la especie $B$. poppei es marcadamente diferente en el largo total y número de huevos lo que probablemente se debe a que esta especie habita en diferente tipo de cuerpos de agua, específicamente lagunas superficiales temporales y permamentes (Menu-Marque et al. 2000; De los Ríos 2008) sin peces (De los RíosEscalante 2010). Esto explicaría la causa que esta especie tenga mayor tamaño y número de huevos en comparación a las otras especies que son propias de lagos y lagunas grandes con peces, (Soto \& Zúñiga 1991; Menu-Marque et al. 2000; De los Ríos \& Soto 2007; De los Ríos 2008) los cuales son depredadores visuales (Gliwicz 2003). En este escenario, Gliwicz (2003), describe que en presencia de depredadores visuales la fecundidad tiende a manifestarse en tamaños corporales relativamente menores como estrategia protectora. No obstante, los resultados de la razón entre el largo total y el número de huevos no revelan diferencias significativas, lo que concuerda con las descripciones de Maly \& Maly (1998), y Othsuka \& Huys (2001), quienes describen una relación constante entre el largo total y los parámetros reproductivos. 


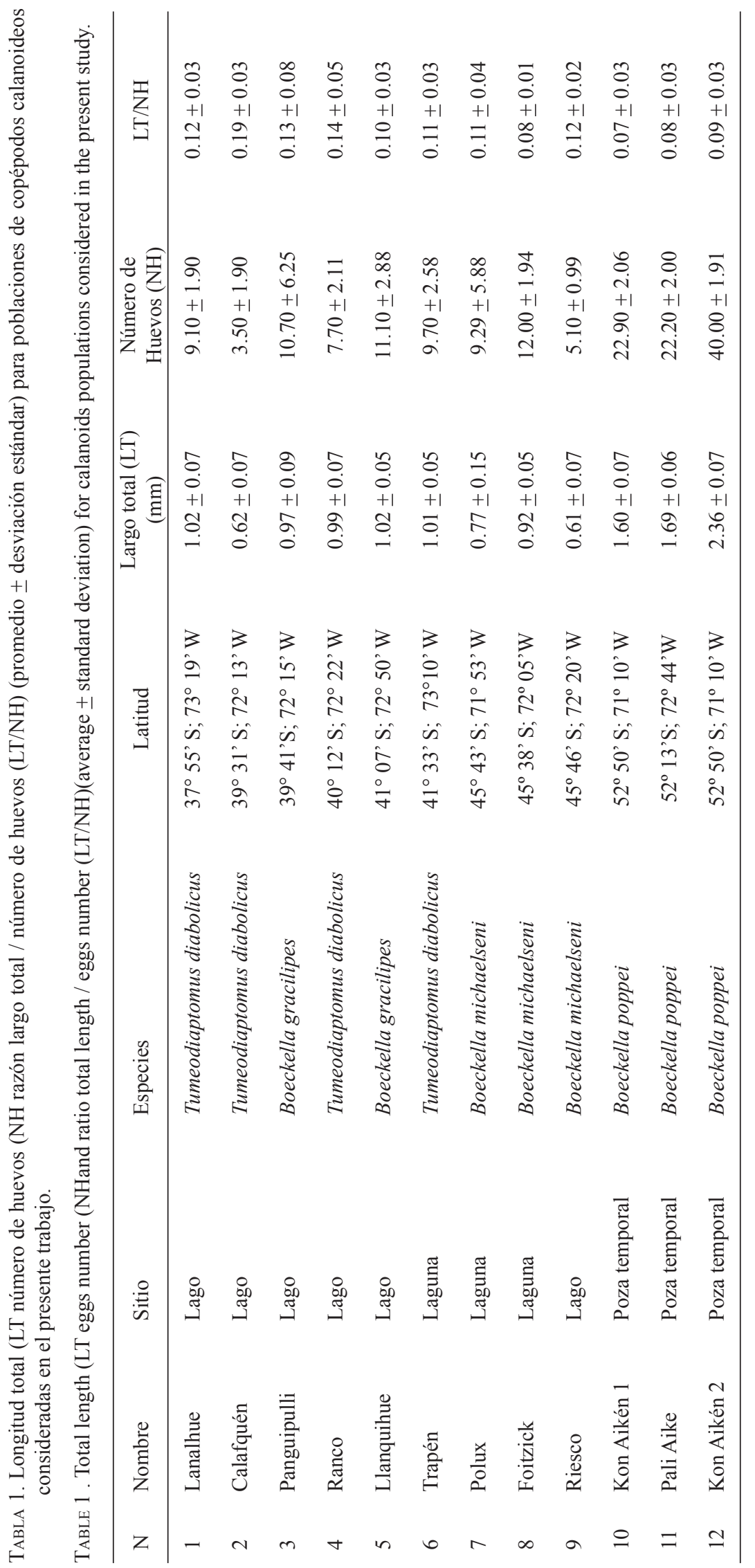


Características reproductivas en Copépodos: Patricio De los Rios-Escalante \& Eriko Carreño

TABla 2. Resultados de los análisis estadísticos de los parámetros considerados en el presente estudio. (n.s = sin diferencias significativas; $*=$ con diferencias significativas).

TABLE 2. Results of statistical analysis for morphometric parameters considered in the present study. $(\mathrm{n} . \mathrm{s}=$ non significant differences; $*=$ significant differences).

\begin{tabular}{|c|c|c|c|}
\hline \multicolumn{4}{|c|}{ COMPARACIÓN ENTRE ESPECIES } \\
\hline & LT & NH & LT/NH \\
\hline & $\begin{array}{l}\mathrm{T}=82.000 \\
\mathrm{P}<0.0001\end{array}$ & $\begin{array}{c}\mathrm{T}=64.0000 \\
\mathrm{P}<0.0001\end{array}$ & $\begin{array}{c}\mathrm{T}=30.0000 \\
\mathrm{P}<0.0001\end{array}$ \\
\hline B. michaelseni - B. gracilipes & * & n.s & n.s \\
\hline B. michaelseni-T. diabolicus & $*$ & n.s & $*$ \\
\hline B. michaelseni - B. poppei & * & * & n.s \\
\hline B. gracilipes - T. diabolicus & n.s & n.s & n.s \\
\hline B. gracilipes - B. poppei & * & * & n.s \\
\hline T. diabolicus - B. poppei & * & * & * \\
\hline \multicolumn{4}{|c|}{ COMPARACIÓN ENTRE POBLACIONES DE CADA ESPECIE } \\
\hline & LT & $\mathrm{NH}$ & $\mathrm{LT} / \mathrm{NH}$ \\
\hline B. michaelseni & $\begin{array}{l}\mathrm{T}=20.000 \\
\mathrm{P}<0.0001\end{array}$ & $\begin{array}{l}\mathrm{T}=16.570 \\
\mathrm{P}<0.0003\end{array}$ & $\begin{array}{l}\mathrm{T}=10.880 \\
\mathrm{P}<0.0043\end{array}$ \\
\hline Polux - Foitzick & n.s & n.s & n.s \\
\hline Polux - Riesco & n.s & * & n.s \\
\hline Foitzick - Riesco & * & * & $*$ \\
\hline B. gracilipes & $\begin{aligned} U & =32.000 \\
P & =0.1780\end{aligned}$ & $\begin{array}{l}\mathrm{U}=47.000 \\
\mathrm{P}=0.8492\end{array}$ & $\begin{array}{l}\mathrm{U}=46.000 \\
\mathrm{P}=0.7600\end{array}$ \\
\hline T. diabolicus & $\begin{array}{l}\mathrm{T}=23.000 \\
\mathrm{P}<0.0001\end{array}$ & $\begin{array}{l}\mathrm{T}=23.000 \\
\mathrm{P}<0.0001\end{array}$ & $\begin{array}{l}\mathrm{T}=13.070 \\
\mathrm{P}=0.0045\end{array}$ \\
\hline Calafquén - Trapén & * & $*$ & * \\
\hline Calafquén - Ranco & * & * & n.s \\
\hline Calafquén - Lanalhue & $*$ & $*$ & $*$ \\
\hline Trapén - Ranco & n.s & n.s & n.s \\
\hline Trapén - Lanalhue & n.s & n.s & n.s \\
\hline Ranco - Lanalhue & n.s & n.s & n.s \\
\hline B. poppei & $\begin{array}{l}\mathrm{T}=11.000 \\
\mathrm{P}<0.0044\end{array}$ & $\begin{array}{l}\mathrm{T}=2.800 \\
\mathrm{P}<0.2480\end{array}$ & $\begin{array}{c}\mathrm{T}=0.02369 \\
\mathrm{P}=0.9882\end{array}$ \\
\hline Kon Aikén 1 - Pali Aike & n.s & n.s & n.s \\
\hline Kon Aikén 1 - Kon Aikén 2 & $*$ & n.s & n.s \\
\hline Pali Aike - Kon Aikén 2 & * & n.s & n.s \\
\hline
\end{tabular}



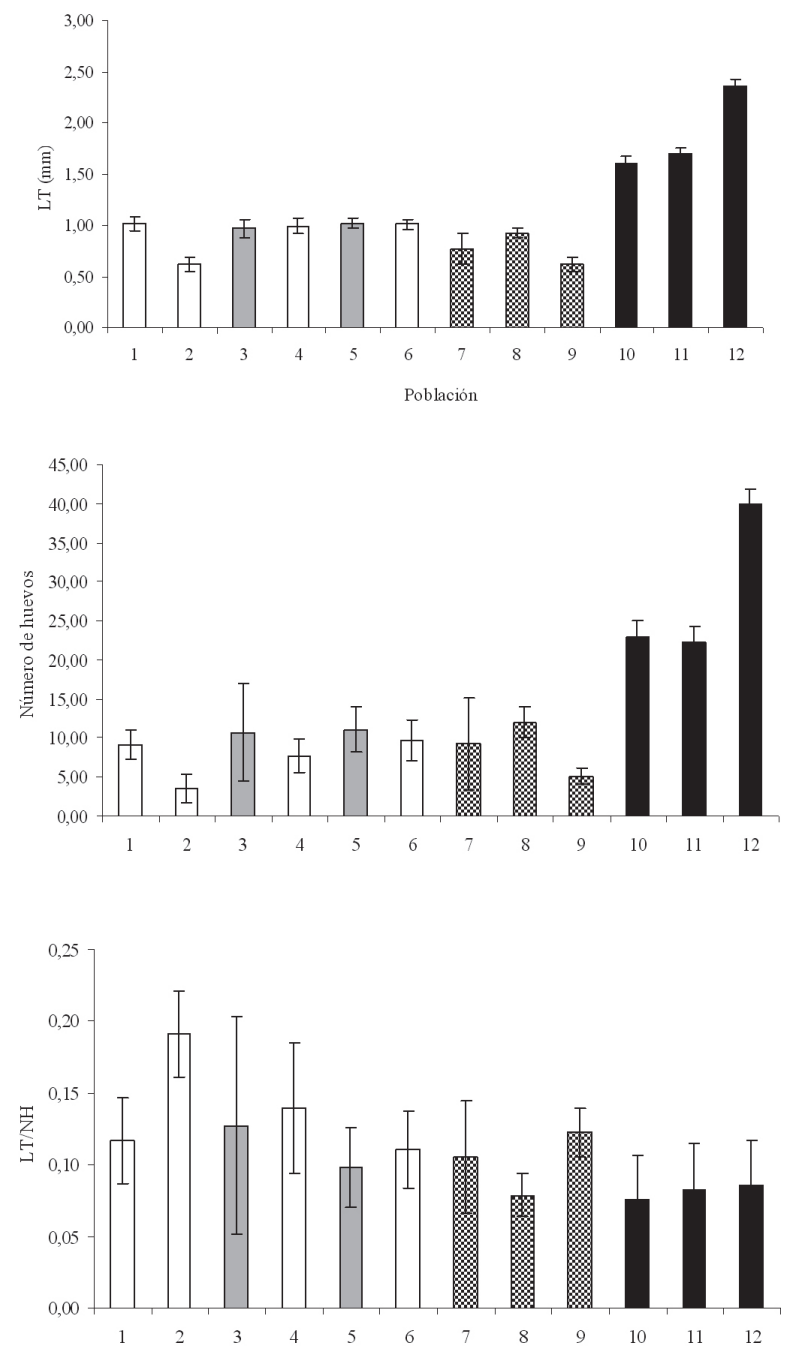

FIGURA 1. Gráficos con los parámetros (promedio + desviación estándar) considerados en el presente estudio. (Ver numeración para cada población en tabla 1).

FIgURE 1. Parameter graphs (average + standard desviation) considered in the present study (See numeration for each population in table 1).

Sobre las diferencias entre las poblaciones de B. michaelseni, revelan la presencia de diferencias en la población del lago Riesco, lo que podría deberse a las condiciones de oligotrofía del sitio en estudio (De los Ríos \& Soto 2007), mientras que los otros sitios restantes son relativamente mesotróficos (Soto et al. 1994; Villalobos 1999). Los resultados para B. gracilipes indican que no hay diferencias significativas lo que se debe a que las poblaciones pertenecen a los denominados "lagos Araucanos" o "lagos nordpatagónicos" que son relativamente similares en sus condiciones limnológicas, específicamente en su condición oligo-mesotrófica (Campos 1984; Villalobos \& Zúñiga 1991; Soto 2002; Woelfl 2007). Una situación diferente sucedería con la especie $T$. diabolicus donde las poblaciones de Ranco, Trapén y Lanalhue fueron similares entre sí, pero diferentes con la del lago Calafquén, a pesar que los lagos Ranco y Calafquén, pertenecen a los lagos Araucanos o Nordpatagónicos que son oligo-mesotróficos (Campos 1984; Soto 2002; Woelfl 2007), mientras que Trapén corresponde a una laguna pequeña localizada en la zona costera cercana a Puerto Montt a los $41^{\circ} \mathrm{S}$, y el lago Lanalhue está localizado en una zona costera a $37^{\circ} \mathrm{S}$ (Tabla 1), ambos grupos de lagos son mesotróficos (De los Ríos 2003). En este contexto, es probable que la disponibilidad de recursos energéticos tenga efectos en la fecundidad de las diferentes poblaciones (Hernández-Trunjillo et al. 2008).

Una situación diferente sucedería con las poblaciones de B. poppei que se incluyeron en el presente estudio, pues pertenecen a lagunas temporales y permanentes (De los Ríos 2008), este tipo de hábitats está expuesto a fuertes variaciones 
ambientales debido a la exposición a la radiación ultravioleta, y evaporación por fuertes vientos (Adamowicz et al. 2007). En este contexto, habría una exposición a agentes estresantes que tendrían efectos sobre el crecimiento poblacional y en consecuencia en la fecundidad que sería afectada (Hall \& Burns 2001a,b). Aunque las muestras de los sitios estudiados se colectaron en periodos de primavera y verano, que corresponden a los periodos de máximas abundancias zooplanctónicas (De los Ríos 2008), las diferencias en el número de huevos para $B$. poppei en comparación a las especies restantes es similar a las descripciones realizadas en copépodos halofílicos en lagos de Nueva Zelanda (Hall \& Burns 2001a,b) lo que se debe a procesos de colonización y extinción.

Los resultados revelan que la fecundidad en copépodos calanoideos serían afectados por las condiciones ambientales específicamente las condiciones tróficas y ambientes extremos.

\section{AGRADECIMIENTOS}

El presente estudio fue financiado por la Dirección de Investigación y Postgrado de la Universidad Católica de Temuco (Fondo para el Desarrollo de la Limnología).

\section{REFERENCIAS}

ARA, K., 2004. Temporal variability and production of the planktonic copepod community in the Canaéia Lagoon Estuarine System, Sao Paulo, Brazil. Zoological Studies 43(2): 179-186.

Adamowicz, S., Menu-Marque, S., Hebert P., \& Purvis, A. 2007. Molecular systematics and patterns of morphological evolution in the Centropagidae (Copepoda: Calanoida) of Argentina. Biological Journal of Linnaean Society of London 90(2): 279-292.

CAmpos, H., 1984. Limnological study of Araucanian lakes (Chile). Verhandlungen International Vereinung Angewaldte Limnolologie 22: 1319-1327.

Daly, M.N., Souissi, S., Yahia-Kéfi, O.D. 2004. Spatial and temporal structure of planktonic copepods in the bay of Tunis (Southwestern Mediterranean Sea). Zoological Studies 43(2): 366-375.

De los Ríos-Escalante, P. 2010. Crustacean zooplankton communities in Chilean inland waters. Crustaceana MONOGRAP HS 12: 1-109.

De los Ríos, P., 2003. Efectos de las disp OnibiUdades de ReCURSos ENERGÉTICOS, ESTRUCTURALES Y DE PROTECCIÓN SOBRE LA DISTRIBUCIÓN Y ABUNDANCIA DE CRUSTÁCEOS ZOOPLANKTÓNICOS lacustres ChILENOS: 1-163. "Tesis Doctoral, Universidad Australde Chile, Facultad de Ciencias.” (Borrar lo que está EN INGIÉS).

De Los Ríos, P., 2008. A null model for explain crustacean zooplankton species associations in central and southern
Patagonian inland waters. Anales del Instituto de la Patagonia 36(1): 25-33.

De los Ríos, P. \& Soto, D. 2007. Crustacean (Copepoda and Cladocera) zooplankton richness in Chilean Patagonian lakes. Crustaceana 80(3): 285-296.

GLIwICZ, Z.M. 2003. Between hazards of starvation and risk of predation: the ecology of offshore animals. International Ecology Institute, Oldendorf / Luhe, Germany. 379 p.

HaLl, C.J. \& BuRNS, C.W. 2001a. Effects of salinity and temperature on survival and reproduction of Boeckella hamata (Copepoda, Calanoida) from a periodically brackish lake. Journal of Plankton Research 23(1): 97-103.

Hall, C.J. \& Burns, C.W. 2001b. Hatching of Boeckella hamata (Copepoda: Calanoida) resting eggs from sediments of a tidally influenced lake. New Zealand Journal of Marine and Freshwater Research 35(2): 235-238.

Hernández-Trujillo, S., Zárate-Villafranco, A., Pacheco-chávez, R., EsquedA-ESCÁRCEGA, G.M., HERnÁNDEZ-AlFONSO, J.R., ACEVES-MEdinA, G. 2008. Variación estacional de la producción de huevos del copépodo calanoideo Centropages furcatus (Dana, 1852) en la Bahía de la Paz, México. Hidrobiológica 18(1): 61-67.

Hirst, A.G. \& Bunker, A.J. 2003. Growth of marine planktonic copepods: global rates and patterns in relation to chlorophyll $a$, temperature and body weight. Limnolology \& Oceanography 48(5): 1988-2010.

Kiorboe, T. 1997.Population regulation and role of mesozooplankton in shaping marine pelagic food webs. Hydrobiologia 363(1/3): 13-27.

Maly, E.J., \& Maly. M.P. 1998. Body size and sexual size sexual dimorphism in calanoid copepods. Hydrobiologia 391(1/3): 173-179

Menu-Marque, S., Morrone, J.J., \& Locascio de Mitrovich, C. 2000. Distributional patterns of the South American species of Boeckella (Copepoda, Centropagidae): a track analysis. Journal of Crustacean Biology 20(2): 262-272.

Oтнsuka S \& Huys, R. 2001. Sexual dimorphism in calanoid copepods: morphology and function. Hydrobiologia 453/454(1): 441-466.

Soto, D. 2002. Oligotrophic patterns in southern Chile lakes: the relevance of nutrients and mixing depth. Revista Chilena de Historia Natural 75(2): 377-393.

Soto, D., Campos, H., Steffen, W., Parra O., \& Zuñiga, L. 1994. The Torres del Paine lake district (Chilean Patagonia): a case of potentially N-limited lakes and ponds. Archiv für Hydrobiologie 99(1/2): 181-197.

Soto, D. \& ZÚÑIGA, L.R. 1991. Zooplankton assemblages of Chilean temperate lakes: a comparison with North American counterparts. Revista Chilena de Historia Natural 64(3): $569-581$.

Villalobos, L. 1999. Determinación de capacidad de carga y balance de fósforo y nitrógeno de los lagos Riesco, Los Palos, y Laguna Escondida en la XI región. Technical Report Fisheries Research Foundation-Chile, FIP-IT/9739.

Villalobos, L \& ZúÑIGA, L.R. 1991. Latitudinal gradient and morphological variability of copepods in Chile: Boeckella gracilipes Daday. Verhandlungen International Vereinung Angewaldte Limnolologie 24: 2834-2838.

Woelfl, S. 2007. The distribution of large mixotrophic ciliates (Stentor) in deep North Patagonian lakes (Chile): First results. Limnologica 37(1): 28-36.

Recibido: 23.11 .11

Aceptado: 29.02 .12 